\title{
Síntese in situ pelo método Pechini para obtenção do compósito SBN61/NFO sem crescimento anormal de grão
}

\section{(In-situ synthesis by Pechini method to obtain SBN61/NFO composite without abnormal grain growth)}

\author{
J.R. Muñoz Hoyos ${ }^{1^{*}}$, E. R. Botero ${ }^{2}$, D. Garcia ${ }^{3}$, R. H. G. A. Kiminami ${ }^{1}$ \\ ${ }^{I} P P G-C E M$, Departamento de Engenharia de Materiais; ${ }^{3}$ Departamento de Física, \\ Universidade Federal de S. Carlos, S. Carlos, SP, Brasil 13565-905 \\ ${ }^{2}$ Universidade Federal de Grande Dourados, Grande Dourados, MS, Brasil 79804-970 \\ *enphysic@hotmail.com,eritonbotero@gmail.com,ducinei.ufscar@gmail.com,ruth@ufscar.br
}

\begin{abstract}
Resumo
Este trabalho teve por objetivo sintetizar in situ pelo método Pechini, pós do compósito magnetoelétrico $\mathrm{Sr}_{0,61} \mathrm{Ba}_{0,39} \mathrm{Nb}_{2} \mathrm{O}_{6} / \mathrm{NiFe}_{2} \mathrm{O}_{4}$ (SBN61/NFO), na proporção 50/50 molar, visando obter amostras sinterizadas com controle de crescimento anormal de grãos da fase SBN61. As amostras de SBN61/NFO foram caracterizadas por análise termogravimétrica, difratometria de raios X, microscopia eletrônica de varredura e transmissão. Refinamento pelo método de Rietveld foi utilizado para quantificar as fases presentes nas amostras sintetizadas e o resultado revelou redução de fases espúrias no compósito SBN61/NFO quando comparado só com o SBN61. O compósito SBN61/NFO após sinterização apresentou densidade relativa acima de 98\%, excelente dispersão das fases NFO e SBN61, baixo grau de percolação da fase NFO e microestrutura sem crescimento anormal de grãos da fase SBN61.
\end{abstract}

Palavras-chave: método Pechini, $\mathrm{Sr}_{0,61} \mathrm{Ba}_{0,39} \mathrm{Nb}_{2} \mathrm{O}_{6} \mathrm{NiFe}_{2} \mathrm{O}_{4}, \mathrm{SBN}_{6}, \mathrm{NiFe}_{2} \mathrm{O}_{4}$.

Abstract

This work involved the in situ synthesis, by the Pechini method, of powders of the magnetoelectric composite $\mathrm{Sr}_{0.61} \mathrm{Ba}_{0.39} \mathrm{Nb}_{2} \mathrm{O}_{6} /$ $\mathrm{NiFe}_{2} \mathrm{O}_{4}(\mathrm{SBN6} 6 / \mathrm{NFO})$, in a 50/50 ratio, to obtain sintered samples with control of abnormal grain growth of the SBN61 phase. The SBN61/NFO samples were characterized by thermogravimetric analysis, $X$-ray diffraction, and scanning and transmission electron microscopy. The phases in the synthesized samples were quantified by the Rietveld refinement method and the result revealed that the SBN61/NFO composite contained fewer spurious phases than the SBN61 phase alone. After sintering, the SBN61/NFO composite showed a relative density of $98.6 \%$, excellent dispersion of the NFO and SBN61 phases, a low degree of percolation of the NFO phase, and microstructure without abnormal grain growth of the SBN61 phase.

Keywords: Pechini method, $\mathrm{Sr}_{0.61} \mathrm{Ba}_{0.39} \mathrm{Nb}_{2} \mathrm{O}_{6} / \mathrm{NiFe}_{2} \mathrm{O}_{4}, \mathrm{SBN61}, \mathrm{NiFe}_{2} \mathrm{O}_{4}$.

\section{INTRODUÇÃO}

Os materiais cerâmicos compósitos magnetoelétricos, constituídos principalmente por uma fase piezoelétrica e a outra magnética, possuemaplicações potenciais em diferentes dispositivos tecnológicos. A resposta magnetoelétrica deste tipo de materiais, principalmente de compósitos particulados (conectividade 0-3), depende do coeficiente piezoelétrico da fase ferroelétrica, do coeficiente magnetostritivo da fase magnética, da adequada homogeneidade entre essas fases, do grau de densificação e da microestrutura do produto final que, por sua vez, vinculam-se a fatores como características do pó e parâmetros de sinterização $[1,2]$. Com relação à fase piezoelétrica, sabe-se que os materiais que apresentam melhor resposta são aqueles que se baseiam no elemento chumbo $(\mathrm{Pb})$. Porém, na atualidade procura-se evitar o uso desses materiais devido a sua alta toxicidade. Entre os materiais piezoelétricos livres de chumbo, o niobato de estrôncio e bário, $\mathrm{Sr}_{\mathrm{x}} \mathrm{Ba}_{1-\mathrm{x}} \mathrm{Nb}_{2} \mathrm{O}_{6}(\mathrm{SBN})$, torna-se um bom candidato para substituir alguns piezoelétricos com chumbo na sua composição, devido a seu coeficiente piezoelétrico relativamente elevado em composições para $\mathrm{x}$ no intervalo entre 0,25 e 0,75 [3-6], que aumenta com o incremento do valor de $\mathrm{x}$. Por outro lado, a ferrita de níquel (NFO) caracteriza-se por apresentar considerável coeficiente magnetostritivo e baixa anisotropia magnética, o que pode favorecer o seu uso em compósitos magnetoelétricos [2].

Entretanto, problemas como o crescimento anormal de grãos e formação de trincas ocorrem durante a sinterização de cerâmicas de SBN. Autores, como [7, 8], propõem que esses problemas estão associados à presença de fases espúrias nos pós, que durante a sinterização conduzem à formação de uma fase rica em $\mathrm{Nb}$ e deficiente em $\mathrm{Ba}$. Com temperatura de fusão inferior à temperatura necessária para densificar as cerâmicas de SBN, essa fase se encontra distribuída em algumas regiões do corpo cerâmico e, ao se tornar líquida durante a sinterização, promove o rápido crescimento dos grãos. Desse modo, reduzir a presença de 
fases espúrias durante a etapa de síntese é fundamental para o controle do crescimento anormal de grãos em cerâmicas de SBN, e por esse motivo métodos químicos como citratogel [9], coprecipitação [10], Pechini [11] e sol-gel [12] têm sido propostos para a obtenção de pós cerâmicos de SBN com elevada pureza e homogeneidade química. Porém, ainda existe dificuldade na sua síntese pela não existência comercial de um reagente de nióbio com alta solubilidade. Acredita-se que o oxalato amoniacal de nióbio produzido pela Companhia Brasileira de Metalurgia e Mineração (CBMM) é uma boa alternativa para seu uso devido à sua fácil solubilidade em água. Foi reportado o sucesso da obtenção pelo método Pechini do $\mathrm{Sr}_{\mathrm{x}} \mathrm{Ba}_{1-\mathrm{x}} \mathrm{Nb}_{2} \mathrm{O}_{6} \operatorname{com} \mathrm{x}=0,33$ sem a presença de fases espúrias utilizando esse reagente, e que com o incremento do valor de $\mathrm{x}$ para 0,50 e 0,73 (SBN com maior coeficiente piezoelétrico) fases espúrias foram detectadas [13]. Contudo, trabalhos realizados na síntese in situ pelo método Pechini dos compósitos cerâmicos 0,9PZN-0,1PT/CFO relatam a obtenção da fase perovskita do $0,9 \mathrm{PZN}-0,1 \mathrm{PT}$ pura e que a presença da fase ferrita de cobalto (CFO) auxiliou na estabilização da fase $0,9 \mathrm{PZN}-$ 0,1PT [14].

Por outro lado, para a produção de compósitos particulados, também é importante garantir uma boa dispersão da fase magnética na fase piezoelétrica. Além disso, sabe-se que quando a concentração da fase magnética é alta e o compósito é sintetizado convencionalmente, cada fase separadamente, existe o aumento do grau de percolação gerando um incremento na condutividade dos compósitos degradando as propriedades magnetoelétricas. Assim, diante disso surgiu a necessidade de se encontrar uma forma de se evitar essa percolação. O método de síntese Pechini convencional consiste na formação de quelatos entre cátions metálicos em solução aquosa com um ácido hidrocarboxílico. A presença do poliálcool (etileno glicol) na solução permite promover uma polimerização entre o citrato do íon metálico e o etileno glicol. O aquecimento em temperatura moderada $\left(100{ }^{\circ} \mathrm{C}\right)$ causa reações de esterificação, dando origem a uma resina polimérica altamente viscosa [15-18]. O polímero formado apresenta grande homogeneidade na dispersão dos cátions metálicos e, após tratamento térmico adequado, a parte orgânica é eliminada, obtendo-se a fase desejada. Por outro lado, a síntese in situ que propõe a preparação do compósito a partir da combinação dos precursores de ambas as fases (magnética e ferroelétrica), desde a primeira etapa de síntese, tem demonstrado ser viável para compósitos particulados, encontrando vantagens principalmente na uniformidade da distribuição das fases, permitindo obter uma cerâmica com microestrutura homogênea de cada uma das fases, bem como distribuição de tamanho de grãos estreita de cada fase. A metodologia in situ pelo método Pechini foi desenvolvida em [19, 20] na obtenção de compósitos magnetoelétricos do sistema $\mathrm{PZT} / \mathrm{Fe}_{2} \mathrm{CoO}_{4}$, demonstrando a sua eficiência quanto à homogeneidade da distribuição das fases e baixo grau de percolação da fase magnética no PZT. Assim, este trabalho teve por objetivo a síntese in situ pelo método Pechini de pós do compósito particulado $\mathrm{Sr}_{0,61} \mathrm{Ba}_{039} \mathrm{Nb}_{2} \mathrm{O}_{6} / \mathrm{NiFe}_{2} \mathrm{O}_{4}$, na proporção $50 / 50$ molar, visando a obtenção de amostras densas com boa dispersão das fases, baixo grau de percolação da fase ferrita e microestruturas sem crescimento anormal de grão da fase SBN61.

\section{MATERIAIS E MÉTODOS}

Para a síntese in situ do sistema $\mathrm{Sr}_{0,61} \mathrm{Ba}_{0,39} \mathrm{Nb}_{2} \mathrm{O}_{6} / \mathrm{NiFe}_{2} \mathrm{O}_{4}$, SBN61/NFO, inicialmente foi misturado o etilenoglicol (Merck) com ácido cítrico (Merck) em $70{ }^{\circ} \mathrm{C}$ até obter uma solução transparente. Separadamente foram dissolvidos os reagentes oxalato amoniacal de nióbio (CBMM), nitrato de estrôncio (Synth), nitrato de bário (Merck), nitrato de níquel (Merck) e nitrato de ferro (Merck) nas quantidades estequiométricas estabelecidas. Essas soluções foram adicionadas na solução de ácido cítrico e etilenoglicol, e o $\mathrm{NH}_{3} \mathrm{OH}$ foi adicionado para levar a solução até $\mathrm{pH} 9$. A solução obtida foi aquecida em uma placa entre 120 e $140{ }^{\circ} \mathrm{C}$ visando eliminar o solvente e formar uma resina polimérica, que posteriormente foi pré-calcinada a $300{ }^{\circ} \mathrm{C}$ durante $12 \mathrm{~h}$ e calcinada em um forno mufla (EDG 3000) entre 900 e $1100{ }^{\circ} \mathrm{C}$ por 2 h. Pó de SBN61 puro também foi sintetizado pelo método Pechini e calcinando a $1250{ }^{\circ} \mathrm{C}$ por $6 \mathrm{~h}$. Os pós cerâmicos obtidos foram caracterizados por análise termogravimétrica (Netzsch STA 409Cell), por medida da area superficial (Micromeritics ASAP 2020), por difração de raios X (Rigaku Rotaflex RU200B com radiação $\mathrm{CuK} \alpha$ ), por microscopia eletrônica de varredura (Philips XL30 FEG) e microscopia eletrônica de transmissão em um microscópio TECNAI. A quantificação das fases presentes em cada amostra foi realizada pelo refinamento dos perfís de difração de raios X pelo método de Rietveld, utilizando o programa GSAS.

Amostras do compósito SBN61/NFO foram analisadas por dilatometria à taxa constante de $10{ }^{\circ} \mathrm{C} / \mathrm{min}$ e isotermas em temperaturas de 1250 e $1300{ }^{\circ} \mathrm{C}$, em um dilatômetro horizontal (Netzsch DIL 402C). Os pós foram conformados em pastilhas cilíndricas com $10 \mathrm{~mm}$ de diâmetro e $2 \mathrm{~mm}$ de espessura por prensagem isostática a $200 \mathrm{MPa}$. As pastilhas conformadas foram sinterizadas em forno tubular Lindberg Blue $\mathrm{M}$ a 1250 e $1300{ }^{\circ} \mathrm{C}$ com taxa de aquecimento de 5 ${ }^{\circ} \mathrm{C} / \mathrm{min}$ e patamar de $3 \mathrm{~h}$. As medidas de densidade aparente das amostras sinterizadas foram realizadas pelo método de imersão pelo princípio de Arquimedes (norma ABNTNBR6620). A caracterização microestrutural foi realizada em um microscópio eletrônico de varredura (MEV) Philips XL-30 FEG. As amostras submetidas à caracterização por MEV foram fraturadas e polidas, e o tamanho médio de grãos foi medido pela técnica linear dos interceptos. Para cada região, foram contados no mínimo 150 grãos, utilizando-se o software Image Pro Plus.

\section{RESULTADOS E DISCUSSÃO}

A Fig. 1 apresenta o resultado da análise por termogravimetria (TG) do pó do compósito SBN61/NFO na 
proporção 50/50 molar sintetizado pelo método Pechini e pré-calcinado a $300{ }^{\circ} \mathrm{C}$ por $12 \mathrm{~h}$. A curva foi dividida em três regiões. Na primeira, entre 100 e $350{ }^{\circ} \mathrm{C}$ observouse uma perda de aproximadamente $5 \%$ de massa devido à evaporação de água. Entre 350 e $700{ }^{\circ} \mathrm{C}$, que correspondeu à segunda região, teve-se a maior perda de massa $(\sim 65 \%)$. Entre 350 e $500{ }^{\circ} \mathrm{C}$, a perda de massa correspondeu à eliminação dos compostos orgânicos durante a formação da fase $\mathrm{NiFe}_{2} \mathrm{O}_{4}$ que, de acordo com a literatura, se forma em temperaturas entre os 400 e $450{ }^{\circ} \mathrm{C}$ para pós obtidos pelo método Pechini [16]. Em $500{ }^{\circ} \mathrm{C}$ teve-se o ponto de inflexão a partir do qual a velocidade de perda de massa foi bem reduzida. Essa redução pode estar associada com a completa formação da fase $\mathrm{NiFe}_{2} \mathrm{O}_{4}$ (NFO). Entre 500 e $700{ }^{\circ} \mathrm{C}$ a perda de massa foi devida à formação dos óxidos primários com os cátions da fase SBN61. Na última região, acima de $700{ }^{\circ} \mathrm{C}$, observou-se perda constante de massa, mas em pequena proporção. Assim, para a calcinação dos pós do compósito foi necessário o controle dos patamares, o primeiro a $450{ }^{\circ} \mathrm{C}$, visando favorecer a formação total da fase NFO, para garantir a sua estabilidade química e reduzir a formação de fases espúrias, com reações indesejadas com os cátions correspondentes à fase SBN61. O segundo patamar foi realizado em temperaturas superiores a $700{ }^{\circ} \mathrm{C}$, visando completar a formação do SBN61.

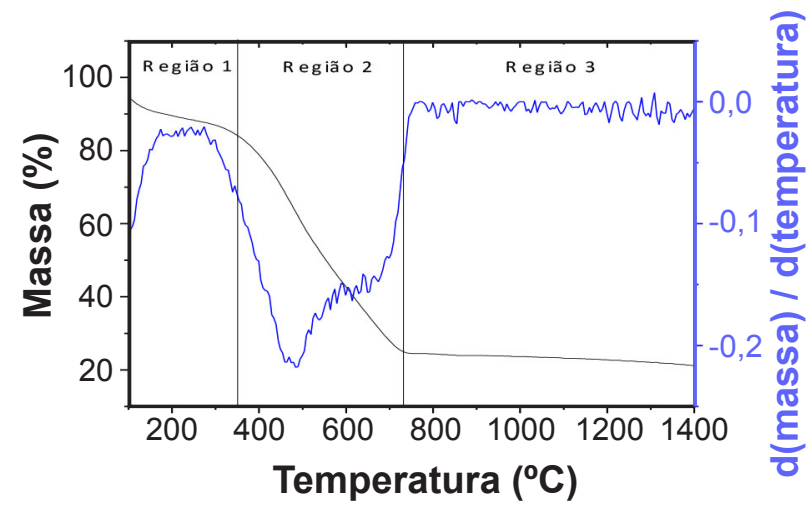

Figura 1: Curvas de termogravimetria (TG) e da derivada (DTG), $\mathrm{d}$ (massa)/dT, de pó de SBN61/NFO 50/50 sintetizado in situ pelo método Pechini e pré-calcinado a $300{ }^{\circ} \mathrm{C} / 12 \mathrm{~h}$.

[Figure 1: Thermogravimetric (TG) and derivative (DTG) curves of the 50/50 SBN61/NFO composite powder synthesized in situ by the Pechini method and pre-calcined at $300{ }^{\circ} \mathrm{C} / 12 \mathrm{~h}$.]

O pó pré-calcinado do compósito SBN61/NFO 50/50, sintetizado in situ pelo método Pechini, foi calcinado entre $900 \mathrm{e}$ $1100^{\circ} \mathrm{C}$ por $2 \mathrm{~h}$, com taxa de aquecimento de $5^{\circ} \mathrm{C} /$ min e patamar intermediário em $450^{\circ} \mathrm{C}$ por $1 \mathrm{~h}$. Os perfis de difração de raios $\mathrm{X}$ das amostras estão apresentados na Fig. 2. Observou-se que foi possível obter as fases SBN61 e NFO a partir de $900{ }^{\circ} \mathrm{C}$, mas tanto nas amostras calcinadas a 900 como a $1000^{\circ} \mathrm{C}$ observou-se um alargamento do pico principal da fase SBN61, em $2 \theta=32^{\circ}$, indicado pela seta na figura, que correspondeu à fase $\mathrm{SrNbO}_{3}$. Por outro lado, em $1100{ }^{\circ} \mathrm{C}$ essa fase espúria parece ter sido eliminada totalmente. Para verificar a existência de fases espúrias nesta temperatura $\left(1100{ }^{\circ} \mathrm{C}\right)$ foi realizado o refinamento do difratograma de raios $\mathrm{X}$ nas condições mínimas exigidas para o refinamento: $2 \theta$ entre $10^{\circ}$ e $90^{\circ}$, passo de $0,02^{\circ}$, tempo de medida de $5 \mathrm{~s}$, filtro de Ni à temperatura ambiente (Fig. 3). A presença do alargamento em $2 \theta=32^{\circ}$ e do pico bem definido em $2 \theta=37^{\circ}$,

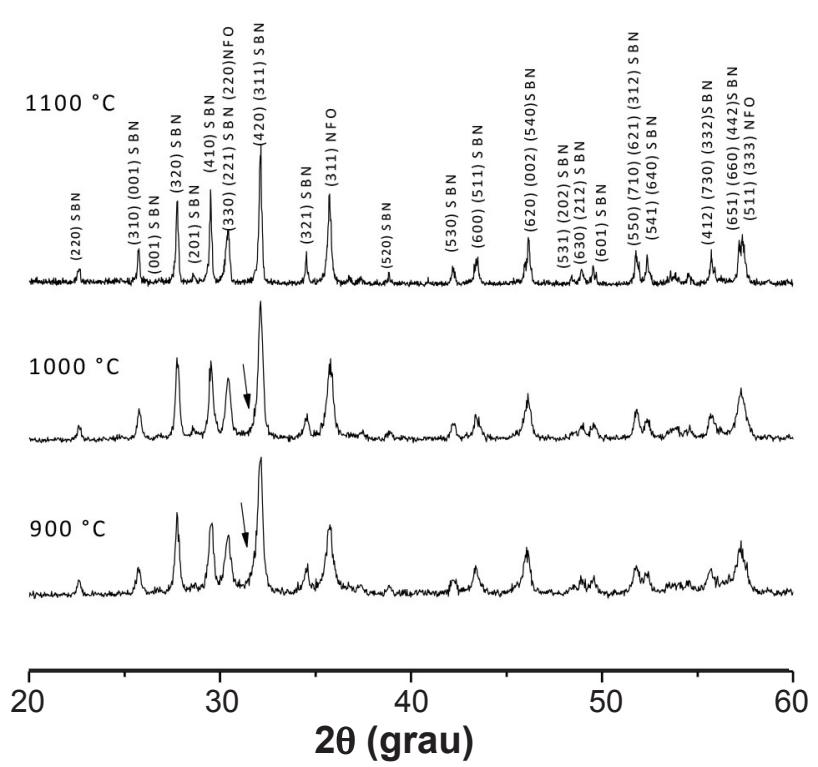

Figura 2: Difratogramas de raios $X$ de amostras de SBN61/NFO 50/50 sintetizadas in situ pelo método Pechini e calcinadas a diferentes temperaturas por $2 \mathrm{~h}$ e com taxa de aquecimento de 5 ${ }^{\circ} \mathrm{C} / \mathrm{min}$. As fases foram identificadas com os arquivos: $\mathrm{NiFe}_{2} \mathrm{O}_{3}$ ICSD-157691; $\mathrm{Sr}_{0,61} \mathrm{Ba}_{0,39} \mathrm{Nb}_{2} \mathrm{O}_{6}$ - ICSD-96013; e $\mathrm{SrNbO}_{3}$ - ICSD42004.

[Figure 2: X-ray diffraction patterns of the 50/50 SBN61/NFO samples synthesized in situ by the Pechini method and calcined at different temperatures with heating rate of $5{ }^{\circ} \mathrm{C} / \mathrm{min}$. The phases were identified with the files: $\mathrm{NiFe}_{2} \mathrm{O}_{4}$ - ICSD-157691; $\mathrm{Sr}_{0.61} \mathrm{Ba}_{0.39} \mathrm{Nb}_{2} \mathrm{O}_{6}-\mathrm{ICSD}-96013$; and $\left.\mathrm{SrNbO}_{3}-\mathrm{ICSD}-42004.\right]$

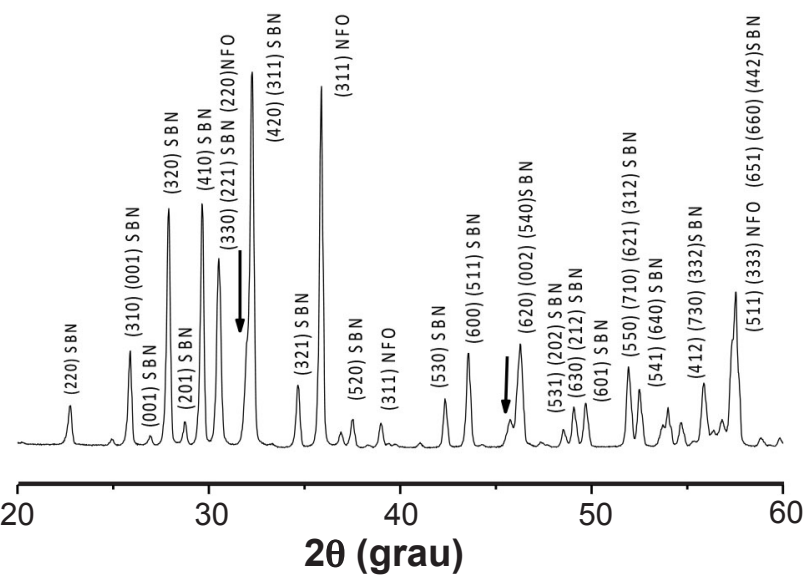

Figura 3: Difratograma de raios $\mathrm{X}$ do compósito SBN61/NFO $50 / 50$ calcinado a $1100{ }^{\circ} \mathrm{C} / 2 \mathrm{~h}$ com aquisição de dados com passo de $0,02^{\circ}$ e tempo de medida de $5 \mathrm{~s}$.

[Figure 3: X-ray diffraction pattern of the 50/50 SBN61/NFO composite calcined at $1100{ }^{\circ} \mathrm{C} / 2$ h obtained with $0.02^{\circ}$ step and acquisition time of $5 \mathrm{~s}$.] 
identificados com setas na figura, foram evidências da existência de traços da fase $\mathrm{SrNbO}_{3}$.

O refinamento do perfil de difração apresentado na Fig. 3 foi realizado até minimizar os valores dos parâmetros de concordância; desse modo $\chi^{2}$ foi 11,1 e Rp e Rwp foram 9,0 e $10,7 \%$, respectivamente. A Fig. 4 apresenta a comparação entre o difratograma calculado pelo refinamento e o experimental, observando-se elevada similaridade entre eles e concordância com os valores dos parâmetros obtidos, permitindo garantir o sucesso do refinamento. As proporções em massa calculadas pelo refinamento da amostra SBN61/NFO na proporção 50/50 foram 52,8 $\pm 0,1 \%$ de $\mathrm{Sr}_{0,61} \mathrm{Ba}_{0,39} \mathrm{Nb}_{2} \mathrm{O}_{6}, 42,0 \pm 0,1 \%$ de $\mathrm{NiFe}_{2} \mathrm{O}_{6}$ e $5,2 \pm 0,1 \%$ de $\mathrm{SrNbO}_{3}$. O refinamento permitiu detectar e quantificar a fase espúria na amostra, não detectada no difratograma de raios X convencional. Ainda com a existência de traços da fase espúria nessa amostra, detectada somente pelo método de Rietveld, acredita-se que o resultado conseguido neste trabalho é muito promissor, pois, de acordo com a literatura [13], conseguir pós de SBN puros é muito complexo e nos trabalhos reportados de síntese desse material puro [9-13] não foram realizados os devidos refinamentos.

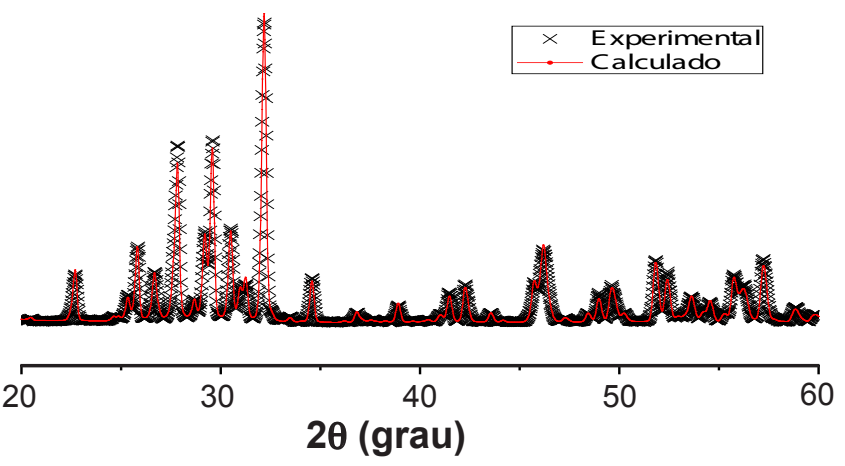

Figura 4: Difratogramas de raios X, experimental e calculado pelo método de Rietveld com GSAS, da amostra SBN61/NFO 50/50. [Figure 4: XRD patterns, experimental and calculated by the Rietveld method with GSAS, of the 50/50 SBN61/NFO sample.]

Para mostrar a dificuldade na obtenção de pós de SBN pelo método Pechini e assim destacar o resultado deste trabalho, foi realizada a síntese individual da fase $\mathrm{Sr}_{0.61} \mathrm{Ba}_{0,39} \mathrm{Nb}_{2} \mathrm{O}_{6}$ (SBN61) por este método, usando dois diferentes tratamentos térmicos na calcinação. A Fig. 5 apresenta os difratogramas de raios X do SBN61; o primeiro tratamento térmico usado foi a $1100^{\circ} \mathrm{C}$ com patamar de $2 \mathrm{~h} \mathrm{e}$ taxa de aquecimento $5^{\circ} \mathrm{C} / \mathrm{min}$. Na Fig. 5a pode-se observar além dos picos correspondentes à fase SBN61 a existência de picos que corresponderam às fases espúrias $\mathrm{Sr}_{2} \mathrm{Nb}_{2} \mathrm{O}_{7} \mathrm{e}$ $\mathrm{BaNb}_{2} \mathrm{O}_{6}$. Visando eliminar as fases espúrias foi aumentada a temperatura e o tempo de calcinação para $1250{ }^{\circ} \mathrm{C}$ e 6 h, respectivamente, mas se observa na Fig. $5 \mathrm{~b}$ que as fases espúrias ainda estão presentes. Foi realizado o refinamento pelo método de Rietveld do perfil de difração de raios $\mathrm{X}$ da amostra de SBN61 calcinada a $1250^{\circ} \mathrm{C}$, até minimizar os valores dos parâmetros de concordância, resultando em $\chi^{2}$ de 6,2 e Rwp e Rp de 9,0 e 7,3\%, respectivamente. A Fig. 6 apresenta a comparação entre o perfil de difração calculado pelo refinamento e o experimental, observandose elevada similaridade entre eles e junto com os valores dos parâmetros de concordância mostraram o sucesso do refinamento. As proporções em massa das fases presentes na amostra calculadas pelo refinamento foram: $64,2 \pm 0,1 \%$ de $\mathrm{Sr}_{0,61} \mathrm{Ba}_{0,39} \mathrm{Nb}_{2} \mathrm{O}_{6}, 29,8 \pm 0,1 \%$ de $\mathrm{Sr}_{2} \mathrm{Nb}_{2} \mathrm{O}_{7}$ e $6,0 \pm 0,2 \%$ de $\mathrm{BaNb}_{2} \mathrm{O}_{6}$, resultando em $35,8 \%$ de fases espúrias.

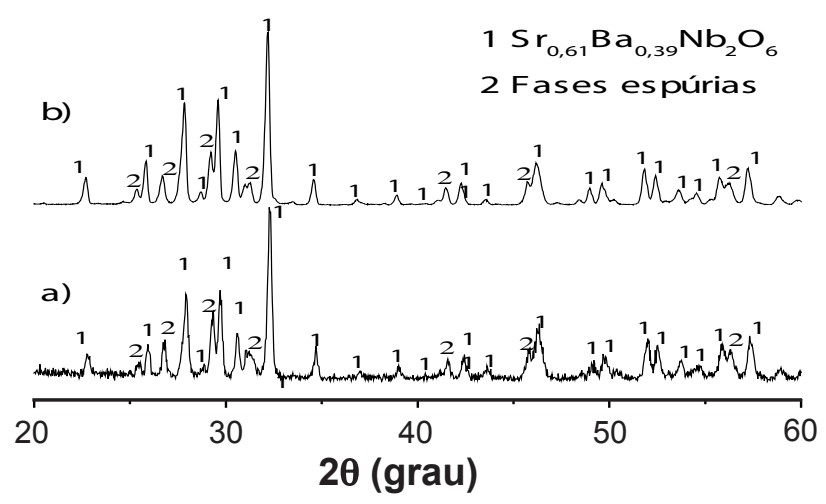

Figura 5: Difratogramas de raios $X$ de amostras de SBN61 sintetizadas pelo método Pechini e calcinadas a: (a) $1100{ }^{\circ} \mathrm{C} / 2 \mathrm{~h}$, e (b) $1250{ }^{\circ} \mathrm{C} / 6 \mathrm{~h}$. As fases foram identificadas com os arquivos: $\mathrm{Sr}_{0.61} \mathrm{Ba}_{0,39} \mathrm{Nb}_{2} \mathrm{O}_{6}$ - ICSD-96013; $\mathrm{Sr}_{2} \mathrm{Nb}_{2} \mathrm{O}_{7}$ - ICSD-281135; e $\mathrm{BaNb}_{2} \mathrm{O}_{6}$ - ICSD-39272.

[Figure 5: X-ray diffraction patterns of SBN61 samples synthesized by the Pechini method and calcined at: (a) $1100{ }^{\circ} \mathrm{C} / 2$ $h$; and (b) $1250{ }^{\circ} \mathrm{C} / 6 \mathrm{~h}$. The phases were identified with the files: $\mathrm{Sr}_{0.61} \mathrm{Ba}_{0.39} \mathrm{Nb}_{2} \mathrm{O}_{6}$ - ICSD-96013; $\mathrm{Sr}_{2} \mathrm{Nb}_{2} \mathrm{O}_{7}-\mathrm{ICSD}-281135$; and $\left.\mathrm{BaNb}_{2} \mathrm{O}_{6}-I C S D-39272.\right]$

Diante dos resultados de quantificação de fases pelo refinamento dos perfis de difração de raios X pelo método de Rietveld do compósito SBN61/NFO e do SBN61, pôde-se observar forte redução da proporção de fases espúrias no total da amostra, sendo 35,8\% na amostra SBN61 e de 5,2\% no compósito SBN61/NFO. Entretanto, é importante salientar que as fases espúrias no compósito foram calculadas com relação à fase SBN61, pois ela teoricamente está constituída principalmente por elementos dessa fase. Desse modo, no compósito o total de fase espúria com respeito à fase SBN61 foi de 9,0\%. Com isso, pôde-se observar que a quantidade de fases espúrias com respeito à fase SBN61 foi reduzida de $35,8 \%$ na amostra SBN61 para 9,0\% no compósito SBN61/ NFO. Tanto na síntese do compósito SBN61/NFO como na síntese individual da fase SBN61, acredita-se que a presença de fases espúrias se deve à formação de precipitados durante a reação em solução líquida, a qual pode comprometer a mobilidade dos cátions, favorecendo a formação de algumas regiões com maior presença de $\mathrm{Sr}^{2+}$, alterando a distribuição atomística aleatória e, consequentemente, dificultando a reação total dos cátions necessária na formação do SBN61 conduzindo à presença das fases espúrias. Desse modo, é possível que a redução de fases espúrias no compósito 


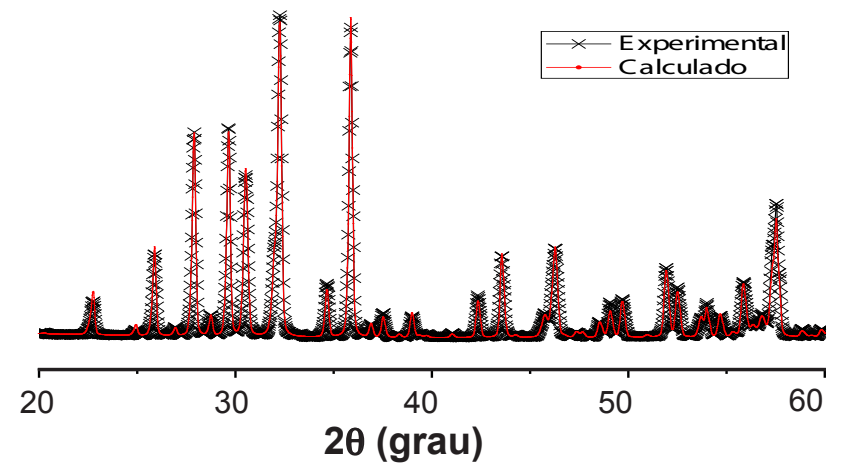

Figura 6: Difratogramas comparativos entre a função experimental e a calculada pelo método de Rietveld com GSAS da amostra SBN61 calcinada a $1250{ }^{\circ} \mathrm{C} / 6 \mathrm{~h}$.

[Figure 6: Comparative diffractograms of the experimental function and the function calculated by the Rietveld method with GSAS of the SBN61 sample calcined at $1250{ }^{\circ} \mathrm{C} / 6 \mathrm{~h}$.]

SBN61/NFO foi devido à presença dos reagentes utilizados na síntese para a formação da fase NFO, que contribuíram indiretamente na redução da quantidade de precipitados formados durante a reação em solução líquida, contribuindo assim na distribuição atomística aleatória de todos os cátions $\left(\mathrm{Sr}^{2+}, \mathrm{Ba}^{2+}, \mathrm{Nb}^{5+}, \mathrm{Fe}^{2+}\right.$ e $\left.\mathrm{Ni}^{2+}\right)$.

O pó do compósito SBN61/NFO na proporção molar 50/50 foi analisado por microscopia eletrônica de varredura (MEV). A Fig. 7 apresenta a micrografia no modo BSE (elétrons retroespalhados) da amostra SBN61/NFO sintetizada in situ e calcinada a $1100{ }^{\circ} \mathrm{C} / 2 \mathrm{~h}$. Foi possível observar a distribuição de tamanhos de partículas e presença de aglomerados. Apesar de conseguir identificar regiões com diferentes contrastes, com a limitada resolução do EDS (espectroscopia por dispersão de energia) no MEV foi difícil definir as fases presentes em cada região, sendo necessária a análise por microscopia eletrônica de transmissão (MET). A Fig. 8 apresenta a micrografia de MET e a quantificação

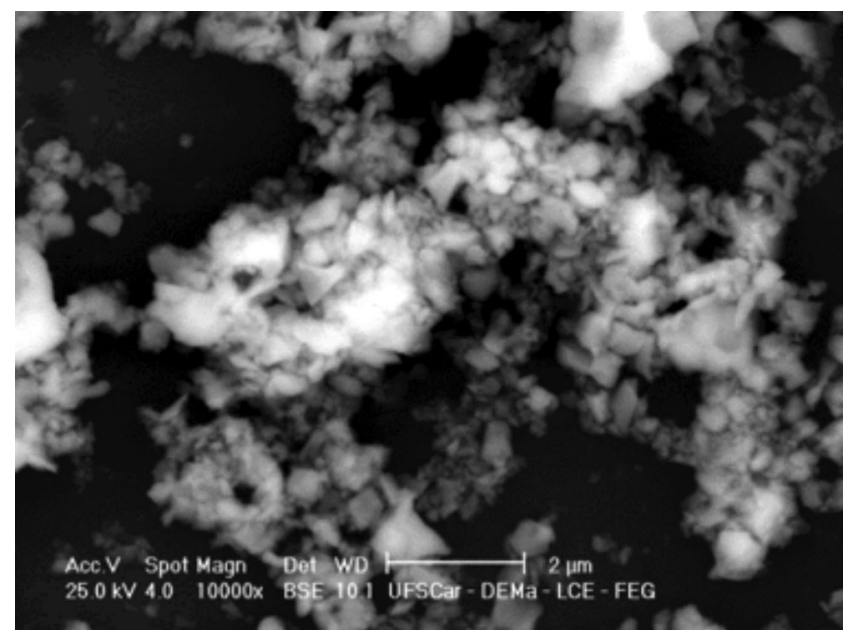

Figura 7: Micrografia obtida por microscopia eletrônica de varredura da amostra SBN61/NFO sintetizada in situ e calcinada a $1100{ }^{\circ} \mathrm{C} / 2 \mathrm{~h}$.

[Figure 7: SEM micrograph of the SBN61/NFO sample synthesized in situ and calcined at $1100{ }^{\circ} \mathrm{C} / 2 \mathrm{~h}$.]
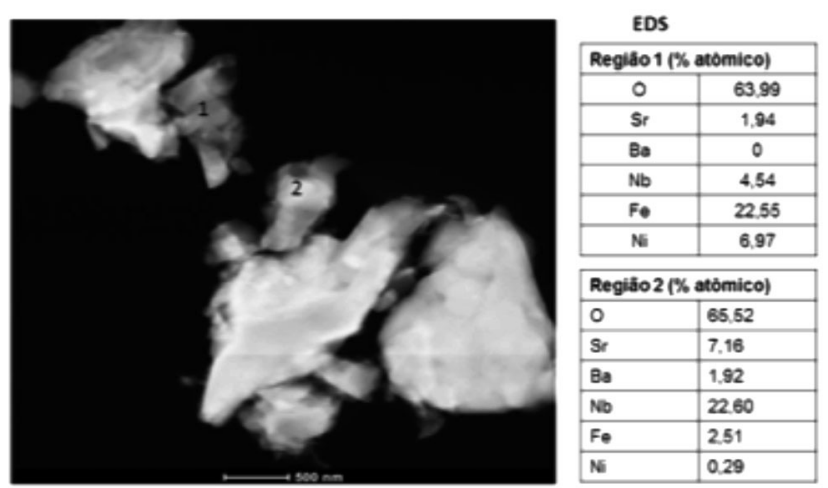

Figura 8: Micrografia obtida por microscopia eletrônica de transmissão da amostra de SBN61/NFO sintetizada in situ pelo método Pechini e calcinada a $1100{ }^{\circ} \mathrm{C} / 2 \mathrm{~h}$, e resultados de EDS das regiões 1 e 2 .

[Figure 8: TEM micrograph of the SBN61/NFO sample synthesized in situ by the Pechini method and calcined at $1100{ }^{\circ} \mathrm{C} / 2 \mathrm{~h}$, and EDS results of regions 1 and 2.]

atômica dos elementos por EDS da amostra SBN61/NFO. Nessa imagem pode-se observar regiões com diferentes tonalidades e, de acordo com os resultados da quantificação por EDS, acredita-se que em zonas, como na região 1 a fase NFO estava presente em maior proporção, e na região 20 SBN61 se encontrava em maior proporção. O valor de área superficial do pó da amostra SBN61/NFO foi de 1,2 $\mathrm{m}^{2} / \mathrm{g}$ e o diâmetro médio de partícula estimado foi de $967 \mathrm{~nm}$, valor associado às partículas secundárias, conforme apresentado na micrografia da Fig. 7, onde se observa que a partícula secundária foi constituída de partículas primárias inferiores a esse valor.

Para o controle da sinterização e do crescimento de grãos, o pó SBN61/NFO foi inicialmente analisado por dilatometria. Inicialmente foi realizada a análise de retração linear à taxa constante de $10{ }^{\circ} \mathrm{C} / \mathrm{min}$ até $1350{ }^{\circ} \mathrm{C}$ (Fig. 9a). Pôde-se observar que a retração da amostra começou em aproximadamente $900{ }^{\circ} \mathrm{C}$ e a temperatura da máxima taxa de retração, que corresponde à temperatura onde a derivada é mínima, foi $1270{ }^{\circ} \mathrm{C}$. Partindo desse resultado decidiu-se analisar o comportamento em função do tempo usando duas isotermas, a primeira em temperatura um pouco inferior, $1250{ }^{\circ} \mathrm{C}$, e a outra um pouco superior, $1300{ }^{\circ} \mathrm{C}$, à temperatura de máxima retração linear por $4 \mathrm{~h}$. Em ambas as temperaturas $\left(1250\right.$ e $\left.1300{ }^{\circ} \mathrm{C}\right)$ observou-se que a retração linear continuou inclusive até depois de $4 \mathrm{~h}$ de aquecimento, e em $1300^{\circ} \mathrm{C}$ a retração foi maior.

Diante dos resultados acima discutidos, novas amostras foram processadas e sinterizadas. Os pós do compósito SBN61/ NFO foram compactados em formato cilíndrico por prensagem isostática a $200 \mathrm{MPa}$ e sinterizados a 1250 e $1300^{\circ} \mathrm{C}$ por $3 \mathrm{~h}$, com taxa de aquecimento de $5^{\circ} \mathrm{C} / \mathrm{min}$. A máxima densificação de $98,6 \pm 1,2 \%$ foi atingida em $1250{ }^{\circ} \mathrm{C}$, e de $97,2 \pm 0,8 \%$ em $1300^{\circ} \mathrm{C}$. Foi calculado o tamanho médio de grãos de cada fase da amostra de SBN61/NFO sinterizada a $1250{ }^{\circ} \mathrm{C}$ (máxima densidade) a partir da micrografia apresentada na Fig. 10c. O tamanho médio de grão da fase SBN61 foi 0,86 $\pm 0,33 \mu \mathrm{m}$, com uma faixa de tamanhos de grão entre 0,44 e 2,42 $\mu \mathrm{m}$, 

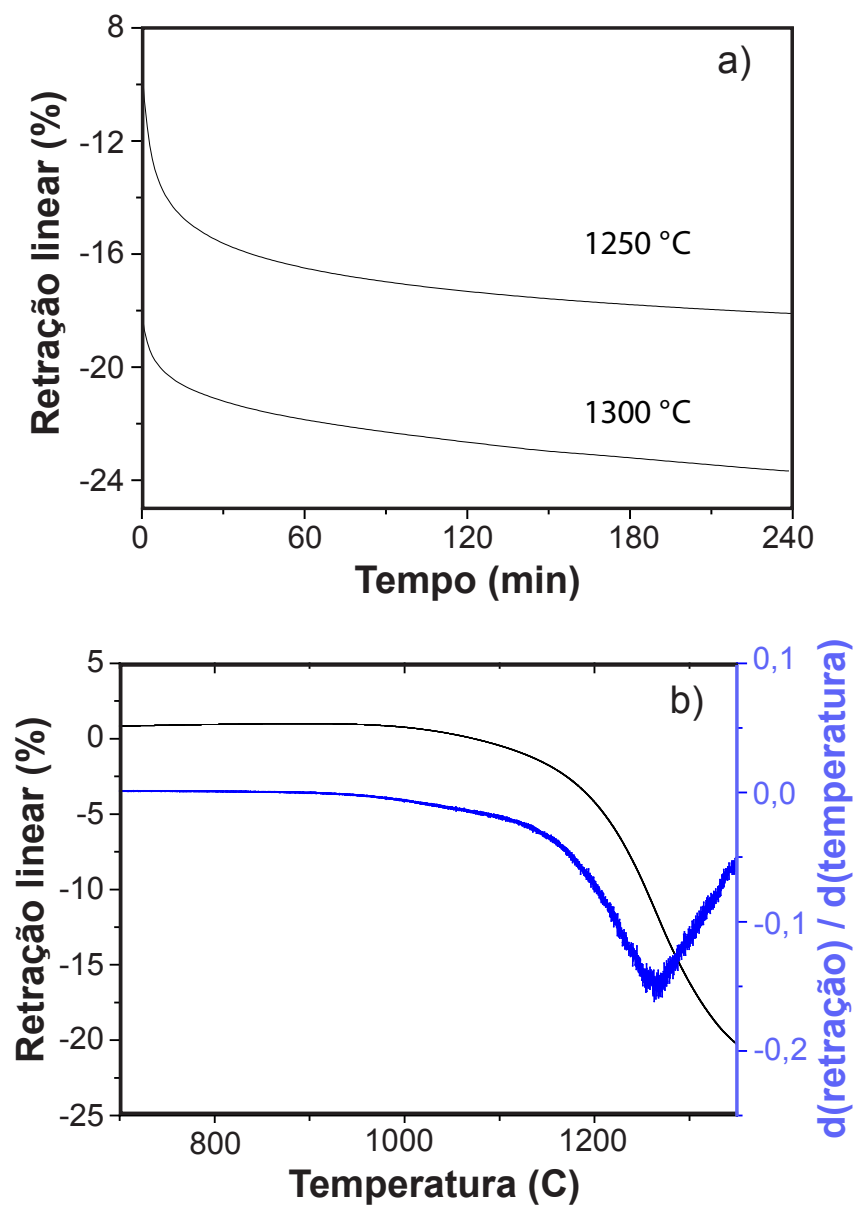

Figura 9: Curvas de retração lineal de amostra de SBN61/NFO: (a) com taxa constante de $10^{\circ} \mathrm{C} / \mathrm{min}$ até $1350{ }^{\circ} \mathrm{C}$; e b) isotermas a 1250 e $1300^{\circ} \mathrm{C}$.

[Figure 9: Linear shrinkage curves of SBN61/NFO samples: (a) at a constant rate of $10{ }^{\circ} \mathrm{C} / \mathrm{min}$; and b) isotherms at 1250 and $\left.1300^{\circ} \mathrm{C}.\right]$

e na fase NFO o tamanho médio foi de 2,31 $\pm 0,76 \mu \mathrm{m}$, com uma faixa entre 1,09 e 2,90 $\mu \mathrm{m}$. Nas micrografias da Fig. 10 foi possível observar a tendência dos grãos da fase SBN61 crescer de forma alongada, mas ainda assim a distribuição de tamanho dos grãos foi estreita sem crescimento exagerado dos grãos, diferente aos reportados na literatura, principalmente em $[7,8]$, que em ambos os casos relatam o crescimento anormal de grãos. Assim, diante desses resultados foi possível evitar o crescimento anormal dos grãos do SBN61 pela redução das fases espúrias na síntese in situ pelo método Pechini e em presença da fase NFO. Patro et al. [21] reduziram o crescimento anormal de grãos em cerâmicas de $\mathrm{Sr}_{\mathrm{x}} \mathrm{Ba}_{1-\mathrm{x}} \mathrm{Nb}_{2} \mathrm{O}_{6} \operatorname{com} \mathrm{x}=0,5$ em amostras sintetizadas pelo método de coprecipitação controlada e sinterização a $1250{ }^{\circ} \mathrm{C}$, com tamanho de grãos na faixa de 10-15 $\mu \mathrm{m}$, o que indicou que comparado com os resultados apresentados neste trabalho houve melhor controle na microestrutura da fase SBN61, pois tanto a faixa de tamanho como o tamanho médio de grãos foi bem inferior, além de ter alcançado densidades muito superiores aos reportados na literatura. Além disso, os resultados deste trabalho foram ainda mais relevantes se for considerado que a fase do SBN foi a
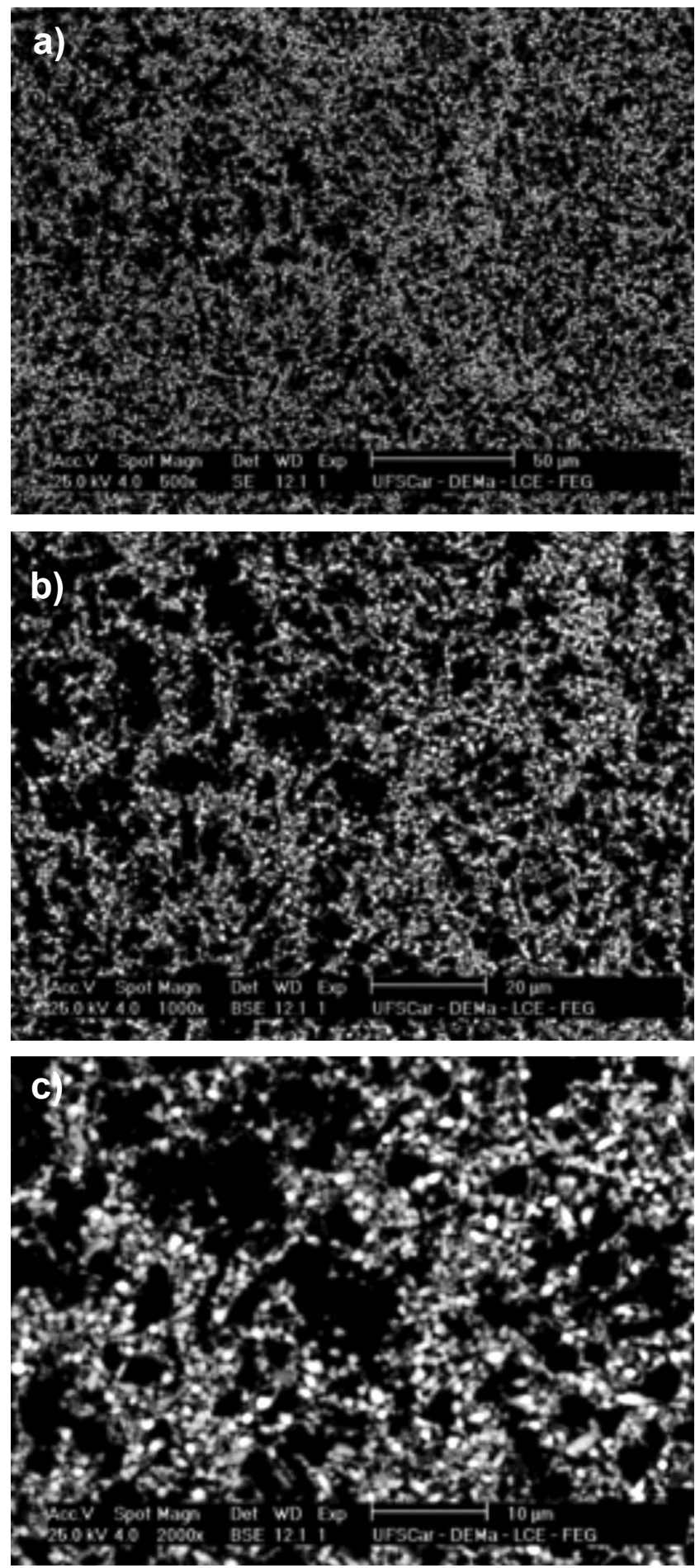

Figura 10: Micrografias obtidas por microscopia eletrônica de varredura com elétrons retroespalhados da amostra de SBN61/ NFO 50/50 sinterizada a $1250{ }^{\circ} \mathrm{C} / 3 \mathrm{~h}$, em diferentes aumentos.

[Figure 10: SEM-BSE micrographs of the 50/50 SBN61/NFO sample sintered at $1250{ }^{\circ} \mathrm{C} / 3 \mathrm{~h}$ with different magnifications.]

correspondente ao valor de $\mathrm{x}=0,61$ do $\mathrm{Sr}_{\mathrm{x}} \mathrm{Ba}_{1-\mathrm{x}} \mathrm{Nb}_{2} \mathrm{O}_{6}$, para o qual é mais difícil o controle do crescimento anormal de grãos. Por outro lado, na fase NFO o crescimento de grãos pode estar associado à temperatura de síntese utilizada para obter o compósito, que foi superior à temperatura necessária para a cristalização da fase pura de NFO, conduzindo a um 
maior tamanho de partículas da fase. Das Figs. 10a e 10b também se pode dizer que houve uma boa dispersão da fase NFO na fase SBN61 e baixo grau de percolação da fase NFO, o que viabiliza o uso da síntese in situ de compósitos SBN61/NFO para garantir uma boa dispersão das fases, controle de crescimento de grãos e densificação nos corpos cerâmicos sinterizados.

\section{CONCLUSÕES}

A síntese in situ pelo método Pechini permitiu obter simultaneamente as duas fases SBN61 e NFO, na proporção molar de 50/50, com traços de fase espúria de $\mathrm{SrNbO}_{3}$ detectada e quantificada pelo refinamento pelo método de Rietveld. Foi possível obter compósito com densidade acima de 98\% com boa dispersão das fases, baixo grau de percolação da fase NFO e sem crescimento anormal de grãos da fase SBN61, viabilizando o uso da síntese in situ para a fabricação de compósitos magnetoelétricos de SBN61/NFO com microestrutura controlada.

\section{AGRADECIMENTOS}

Os autores agradecem à Fundação de Amparo à Pesquisa do Estado de São Paulo (FAPESP) pelo apoio financeiro (Processos n. 2008/04025-0 e 2012/24025-0), à Coordenação de Aperfeiçoamento de Pessoal de Nível Superior (CAPES) pela bolsa concedida, ao projeto PROCAD 2013, auxílio 3012/2014, e à Companhia Brasileira de Metalurgia e Mineração (CBMM), pela doação do oxalato amoniacal de nióbio.

\section{REFERÊNCIAS}

[1] A.J. Moulson, J. M. Herbert, Electroceramics, $2^{\text {nd }}$ Ed., John Wiley \& Sons, Chichester (2003) 97.

[2] J. Ryu, A.V. Carazo, K. Uchino, H. Kim, J. Electroceram. 7 (2001) 17.

[3] R.R. Neurgaonkar, J.R. Oliver, W. K. Cory, L.E. Cross, D. Viehland, Ferroelectr. 160, 1 (1994) 265.

[4] Y.J. Li, X.M. Chen, Y.Q. Lin, Y.H. Tang, J. Eur. Ceram. Soc. 26 (2006) 2839.

[5] T.R. Volk, V. Yu.Salobutin, L.I. Ivleva, N.M. Polozkov,
R. Pankrath, M. Woehlecke, Phys. Solid State 42, 11 (2000) 2129.

[6] T.S. Chernaya, M.O. Marychev, V.A. Ivanov, N.J. Ivanov, E.V. Huprunov, L.I. Ivleva, V.I. Simonov, Crystallogr. Rep. 52, 6 (2007) 1056.

[7] H. Lee, R. Freer, J. Mater. Sci. 33 (1998) 1703.

[8] M.S. Kim, J.H. Lee, J.J. Kim, H.Y. LEE, S.H. Cho, Japan Soc. Appl. Phys. 41 (2002) 7048.

[9] D. Wu, W. Gong, H. Dengand, M. Li, J. Phys. D: Appl. Phys. 40 (2007) 5002.

[10] S. Prasad, J. Gajbhiye, J. Alloys Comp. 265, 1-2 (1998) 87.

[11] A.B. Panda, A. Pathak, P. Pramanik, Bull. Mater. Sci. 25, 2-3 (2002) 501.

[12] B. Domenichini, T. Caillot, Acta Mater. 51, 16 (2003) 4815.

[13] S. Gaona, J.R. Muñoz, C.F.V. Raigoza, A. Ramirez, A. Caneiro, Bol. Soc. Esp. Ceram. Vidr. 52 (2013) 291.

[14] C.P. Fernandez, "Síntese in situ pelo método Pechini e sinterização por métodos não convencionais de compósitos magnetoelétricos particulados", Tese Dr., Univ. Fed. S. Carlos, S. Carlos (2015).

[15] J.R. Muñoz Hoyos, F.L. Zabotto, D. Garcia, R.H.G.A. Kiminami, Cerâmica 59, 351 (2013) 360.

[16] J.R. Muñoz Hoyos, "Síntese, sinterização em forno de micro-ondas e caracterização da $\mathrm{Fe}-\mathrm{Ni}$, PZT e do sistema multifuncional magnetoelétrico PZT/Fe-Ni", Diss. Mestr., Univ. Fed. S. Carlos, S. Carlos (2012).

[17] G. Mu, S. Yang, J. Li, M. Gu, J. Mater. Process. Technol. 182 (2007) 382.

[18] M.P. Pechini, "Barium titanium citrate, barium titanate and processes for producing same", U.S. Patent 3231328 A, 1966.

[19] P.C.P. Fernández, "Síntese, sinterização e caracterização de nanocompósitos magnetoelétricos particulados de PZT/ Fe-Co", Diss. Mestr., Univ. Fed. S. Carlos, S. Carlos (2011). [20] C.P.F. Perdomo, R.H.G.A. Kiminami, F.L. Zabotto, D. Garcia, In: Processing and Properties of Advanced Ceramics and Composites VI, Ceramic Transactions, John Wiley \& Sons, Hoboken 249 (2014) 27.

[21] P.K. Patro, A.R. Kulkarni, C.S. Harendranath, J. Eur. Ceram. Soc. 23 (2003) 1329.

(Rec.02/10/2016, Rev. 03/01/2017, Ac. 18/02/2017) 